\title{
RADIAL GROWTH OF FUNCTIONS IN THE KORENBLUM SPACE
}

\author{
A. BORICHEV, YU. LYUBARSKII, E. MALINNIKOVA, AND P. THOMAS
}

Dedicated to Victor Petrovich Havin on the occasion of his 75th birthday

\begin{abstract}
The radial behavior of analytic and harmonic functions that admit a certain majorant in the unit disk is studied. We prove that the extremal growth or decay may occur only along small sets of radii and give precise estimates for these exceptional sets.
\end{abstract}

\section{§1. INTRODUCTION}

We study the radial behavior of analytic and harmonic functions in the unit disk. In order to describe the problem, we recall the classical results of Lusin and Privalov (see, e.g., [12, Chapter IV]).

Theorem A (Lusin, Privalov). Let $f(z)$ be a function analytic in the unit disk $\mathbf{D}$, and let $E$ be a subset of the unit circle $\mathbf{T}$ of positive linear measure. If $f$ tends to zero nontangentially at each point of $E$, then $f=0$.

The situation changes if one considers radial limits.

Theorem $\mathbf{B}$ (Lusin, Privalov). There exists an analytic function $f$ in $\mathbf{D}$ such that $\lim _{r \rightarrow 1} f\left(r e^{i \phi}\right)=0$ for almost every $\phi \in[0,2 \pi)$.

These results can be reformulated for harmonic functions. The first theorem says that there are no $u \in \operatorname{Harm}(\mathbf{D})$ that tend to $+\infty$ nontangentially on a set of positive measure in $\mathbf{T}$, while the second gives a function in $\operatorname{Harm}(\mathbf{D})$ that tends radially to $+\infty$ almost everywhere on $\mathbf{T}$ (we remark that the function $f$ in Theorem B can be chosen to be zero-free). We refer also to [1, 9] for other relevant examples. The growth of harmonic functions tending radially to $+\infty$ almost everywhere can be arbitrarily slow: the statement below is a special case of a theorem in $[9]$.

Theorem $\mathbf{C}$ (Kahane, Katznelson). Let $v(r)$ be a positive and monotone nondecreasing function on $[0,1)$ with $\lim _{r \rightarrow 1-} v(r)=\infty$. Then there exists $u \in \operatorname{Harm}(\mathbf{D})$ such that

$$
u(z) \leq v(|z|)
$$

and $\lim _{r \rightarrow 1-} u\left(r e^{i \phi}\right)=\infty$ for a.e. $\phi \in[0,2 \pi)$.

2010 Mathematics Subject Classification. Primary 30J99, 31A20.

Key words and phrases. Spaces of analytic functions in the disk, harmonic functions, boundary values, Korenblum spaces.

A. B. was partially supported by the ANR project DYNOP.

Yu. L. was partly supported by the Research Council of Norway, grants 160192/V30 and 177355/V30.

E. M. was partly supported by the Research Council of Norway, grants 160192/V30 and 177355/V30. 
In this paper we address the following questions.

Let the function $v$ be as above, and let $u \in \operatorname{Harm}(\mathbf{D})$ satisfy (1.1).

- How fast (with respect to $v$ ) can u grow to $+\infty$ along massive sets of radii?

- How fast (with respect to $v$ ) can u decay to $-\infty$ along massive sets of radii?

We restrict ourselves to a particular majorant function

$$
v(r)=\log \frac{1}{1-r} .
$$

This choice is motivated by its relevance to the classical Korenblum space $A^{-\infty}$ (see [10]). It also serves as a model case for more general majorants.

A typical answer to the first question is that at almost all radii the function $u$ grows (if it grows at all) more slowly than $v$, with the exceptional set having zero Lebesgue measure. We give precise estimates for the size of exceptional sets in terms of the Hausdorff measures with respect to the scale of functions $h_{\alpha}(t)=t|\log t|^{\alpha}, \alpha>0$.

Regarding the second question, first we remark that a function $u$ that satisfies (1.1) may decay to $-\infty$ along radii much faster than $-v(r)$, so that the harmonic function $-u$ may fail to satisfy (1.1). However, for a given $M(s), s>0$, such that $M(s) / s \rightarrow+\infty$ as $s \rightarrow+\infty$, the set $\{z \in \mathbf{D}: u(z)<-M(v(|z|))\}$ is small (sharp estimates for typical $M$ were obtained in [3]). We show that along most radii $-u$ grows slower than $v$, the estimates of the exceptional set being the same as in the answer to the first question. For the maximal possible decay of harmonic functions throughout the whole disk, see [11, 2] and the references therein.

Our statements can be reformulated for zero-free functions belonging to the Korenblum class. Now the second statement describes how fast an analytic function can approach zero along some radii. Actually this statement holds true for any (not necessarily zerofree) function in $A^{-\infty}$. At the same time, adding zeros may result in extremal radial growth along almost all radii.

The paper is organized as follows. The next section includes definitions and formulations of the main results in terms of analytic functions. In $\S 3$ we deal with harmonic (subharmonic) functions. By using standard estimates of the Poisson integral, we show that fast radial growth (decay) implies nontangential growth (decay), and thus, may occur only on a set of zero measure.

The main results are proved in $\S 4$ : departing from nontangential growth (decay) and harmonic measure inequalities, we obtain more precise estimates of the size of the exceptional sets. These estimates are sharp, as shown by examples collected in $\S 5$. We also give an example showing that the situation becomes very different if one considers growth merely along sequences of points: there exists a function such that every radius contains a sequence of points of extremal growth. In $\S 6$ we consider positive harmonic functions satisfying (1.1) and show that for such functions the answer to the first question is different. Finally, $\S 7$ contains a theorem about Hausdorff measure of Cantor-type sets.

\section{$\S 2$. Formulation of the Main Results}

An analytic function $f$ in $\mathbf{D}$ is said to be of class $A^{-\infty}$ if there exist constants $C$ and $k$ such that

$$
|f(z)| \leq \frac{C}{(1-|z|)^{k}}
$$


For a function $f \in A^{-\infty}$, we define

$$
\begin{aligned}
& D_{+}(f)=\left\{\theta \in[0,2 \pi): \liminf _{r \rightarrow 1} \frac{\log \left|f\left(r e^{i \theta}\right)\right|}{|\log (1-r)|}>0\right\}, \\
& D_{-}(f)=\left\{\theta \in[0,2 \pi): \limsup _{r \rightarrow 1} \frac{\log \left|f\left(r e^{i \theta}\right)\right|}{|\log (1-r)|}<0\right\} .
\end{aligned}
$$

We recall the definition of the Hausdorff measure. Given an increasing function $\lambda$, $\lambda:[0,1) \rightarrow[0,+\infty), \lambda(0)=0$, we denote by $H_{\lambda}(C)$ the corresponding Hausdorff measure of a set $C \subset \mathbf{T}$ (or $C \subset \mathbf{R}$ ), defined as

$$
H_{\lambda}(C)=\liminf _{\epsilon \rightarrow 0}\left\{\sum_{s} \lambda\left(\left|J_{s}\right|\right): C \subset \bigcup_{s} J_{s},\left|J_{s}\right|<\epsilon\right\},
$$

where the $J_{s}$ are $\operatorname{arcs}$ of $\mathbf{T}$ (respectively, intervals of $\mathbf{R}$ ).

The main results of the paper give estimates on the size of the sets $D_{ \pm}(f)$.

Theorem 1. Let $\lambda(t)=o\left(t|\log t|^{\omega}\right), t \rightarrow 0$, for every $\omega>0$. Then

(i) $H_{\lambda}\left(D_{+}(f)\right)=0$ for each zero-free $f \in A^{-\infty}$;

(ii) $H_{\lambda}\left(D_{-}(f)\right)=0$ for each $f \in A^{-\infty}$.

The next statement shows that these results are sharp.

Proposition 1. For any $\alpha>0$ there exists a zero-free function $f \in A^{-\infty}$ such that $f^{-1} \in A^{-\infty}$ and $H_{\lambda}\left(D_{+}(f)\right)=\infty$ for $\lambda(t)=t|\log t|^{\alpha}$.

Note that for zero-free functions we have $D_{+}(f)=D_{-}\left(f^{-1}\right)$; thus, Proposition 1 shows that our condition on $\lambda$ is sharp in both assertions of Theorem 1 .

There are no analogs of the first estimate in Theorem 1 for general functions of class $A^{-\infty}$. This can be seen by analyzing functions having "regular" growth in $\mathbf{D}$ such as those given in Theorem 2 in 13. The argument in 13 . relies on atomization techniques, and in this paper we use a simple explicit function constructed by Horowitz.

Given a number $\mu>1$ and an integer $\beta>1$, consider the function

$$
f_{\mu, \beta}(z)=\prod_{k=1}^{\infty}\left(1+\mu z^{\beta^{k}}\right) .
$$

The results of $\left[8\right.$ (see also [10]) show that $f_{\mu, \beta} \in A^{-\infty}$.

Proposition 2. Let $\mu$ and $\beta$ be numbers satisfying

$$
1-\frac{1}{\mu^{1-1 / \sqrt{\beta}}} \geq \frac{1}{e}, \quad \mu>e .
$$

Then $D_{+}\left(f_{\mu, \beta}\right)$ has full measure in $\mathbf{T}$.

We also consider the extremal growth on subsets of radii. Given a function $f \in A^{-\infty}$, we denote

Clearly, $G_{+}(f) \supset D_{+}(f)$.

$$
G_{+}(f)=\left\{\theta \in[0,2 \pi): \limsup _{r \rightarrow 1} \frac{\log \left|f\left(r e^{i \theta}\right)\right|}{|\log (1-r)|}>0\right\} .
$$

Proposition 3. There exists a zero-free $f \in A^{-\infty}$ such that $G_{+}(f)=\mathbf{T}$.

The estimate in the first statement in Theorem 1 can be improved if we assume that $|f|$ is bounded from below by a positive constant. This improvement corresponds to the difference between estimates of the Poisson integral with respect to a premeasure (as in Theorem 11) and a Borel measure as in Theorem 2 below; we refer the reader to 10 for the definition and basic properties of premeasures. 
Theorem 2. Let $\lambda(t)=t|\log t|$. Suppose that $f \in A^{-\infty}$ and $|f|$ is bounded from below by a positive constant. Then the set $G_{+}(f)$ is a countable union of sets with finite $H_{\lambda^{-}}$ measure.

There exists $f \in A^{-\infty}$ such that $|f|$ is bounded from below by a positive constant and $H_{\lambda}\left(D_{+}(f)\right)=\infty$.

In order to construct examples in Proposition 1 and Theorem 2, we use Cantor-type sets having the following structure:

$$
C=\bigcap_{s} C_{s}, \quad C_{s} \supset C_{s+1}, \quad C_{0}=[0,1],
$$

where each set $C_{s}$ is a union of $N_{s}$ segments $\left\{I_{j}^{(s)}\right\}_{j}$ of the same length $l_{s}$. For each such segment the intersection $C_{s+1} \cap I_{j}^{(s)}$ is a union of $k_{s}$ disjoint segments of length $l_{s+1}$. Of course, we assume that

$$
l_{s} \searrow 0, s \rightarrow \infty ; \quad k_{s} l_{s+1}<l_{s}, \text { and } N_{s}=k_{0} k_{1} \cdots k_{s-1} .
$$

Theorem 3. Let $\lambda:[0,1) \rightarrow[0,+\infty)$ be a continuous and monotone increasing function such that $\lambda(0)=0$ and for some $a>0$ and $s>s_{0}$ we have

$$
\frac{\lambda(l)}{l} \geq a \frac{\lambda\left(l_{s+1}\right)}{l_{s+1}} \quad \text { for any } \quad l \in\left[l_{s+1}, l_{s}\right) .
$$

Then

$$
\liminf _{s \rightarrow \infty} N_{s} \lambda\left(l_{s}\right) \geq H_{\lambda}(C) \geq \frac{a}{2} \liminf _{s \rightarrow \infty} N_{s} \lambda\left(l_{s}\right) .
$$

Other results of this type were given in [5, 4]; unfortunately, we are not able to apply those results in our situation. We prove Theorem 3 in the last section and believe that it may be of its own interest.

\section{§3. From radial to nontangential growth}

To deal with zero-free functions of class $A^{-\infty}$, we consider the corresponding class of harmonic functions. A function $u \in \operatorname{Harm}(\mathbf{D})$ is said to be of class $\mathcal{K}$ if there exists a constant $C$ such that

$$
u(z) \leq C \log \frac{e}{1-|z|}, \quad z \in \mathbf{D} .
$$

If $f \in A^{-\infty}$ is a zero-free function, then $u(z)=\log |f(z)|$ belongs to $\mathcal{K}$.

Given a function $u \in \mathcal{K}$, we denote

$$
\begin{aligned}
& E_{+}(u)=\left\{\theta \in[0,2 \pi): \liminf _{r \rightarrow 1} \frac{u\left(r e^{i \theta}\right)}{|\log (1-r)|}>0\right\}, \\
& E_{-}(u)=\left\{\theta \in[0,2 \pi): \limsup _{r \rightarrow 1} \frac{u\left(r e^{i \theta}\right)}{|\log (1-r)|}<0\right\} .
\end{aligned}
$$

In this section we show that fast radial growth along radii implies fast nontangential growth. We use the standard notation

$$
P\left(r e^{i \theta}\right)=\frac{1}{2 \pi} \frac{1-r^{2}}{1-2 r \cos \theta+r^{2}}
$$

for the Poisson kernel.

Suppose $r \in(0,1), \tau \in(0,1)$, and $0<\delta<\tau(1-r)$. Then

$$
P\left(r e^{i \theta}\right)>(1-\tau) P\left(r e^{i(\theta+\delta)}\right) .
$$

This inequality can be proved by elementary calculations; also, it can be viewed as a special case of the Harnack inequality. 
Lemma 1. Let $u \in \mathcal{K}$, and let $C=\sup \left\{u(z)(\log e /(1-|z|))^{-1}: z \in \mathbf{D}\right\}$. Suppose that for some $\sigma>0, \theta \in[0,2 \pi]$, and $r \in(0,1)$ we have

Then

$$
u\left(r e^{i \theta}\right)>\sigma \log \frac{e}{1-r} .
$$

$$
u\left(r e^{i(\theta+\delta)}\right)>\frac{\sigma}{2} \log \frac{e}{1-r}
$$

for $|\delta|<\tau_{1}(1-r)$, where $\tau_{1}=\tau_{1}(C, \sigma)>0$.

Proof. Let $R=(1+r) / 2$. We apply (3.1), replacing $P(r, \cdot)$ by $P(r / R, \cdot)$ and assuming that $|\delta|<\tau\left(1-\frac{r}{R}\right)$ with $\tau<1$. We obtain

$$
\begin{aligned}
u\left(r e^{i \theta}\right) & =\int_{0}^{2 \pi} u\left(R e^{i \phi}\right) P\left(\frac{r}{R} e^{i(\theta-\phi)}\right) d \phi \\
& =(1-\tau) u\left(r e^{i(\theta+\delta)}\right)+\int_{0}^{2 \pi} u\left(R e^{i \phi}\right)\left(P\left(\frac{r}{R} e^{i(\theta-\phi)}\right)-(1-\tau) P\left(\frac{r}{R} e^{i(\theta+\delta-\phi)}\right)\right) d \phi \\
& <(1-\tau) u\left(r e^{i(\theta+\delta)}\right)+C \tau \log \frac{e}{1-R} .
\end{aligned}
$$

Hence,

$$
\sigma \log \frac{e}{1-r}<(1-\tau) u\left(r e^{i(\theta+\delta)}\right)+C \tau \log \frac{e}{1-r}+C \tau \log 2 .
$$

Taking $\tau$ sufficiently small, we obtain relation (3.2) with $\tau_{1}=\tau / 2$.

The proof of Lemma 1 works also for the radial decay; however, for this case we need a more general setting involving subharmonic functions.

Lemma 2. Let $v$ be a subharmonic function on $\mathbf{D}$, and let

$$
v(z) \leq C \log \frac{e}{1-|z|}, \quad z \in \mathbf{D}
$$

Suppose that

$$
v\left(r e^{i \theta}\right)<-\sigma \log \frac{e}{1-r}, \quad r>r_{0}
$$

for some $\sigma>0, \theta \in[0,2 \pi]$, and $r_{0} \in(0,1)$. Then

$$
v\left(r e^{i(\theta+\delta)}\right)<-\frac{\sigma}{2} \log \frac{e}{1-r}, \quad r>r_{1},
$$

for $|\delta|<\tau_{2}(1-r)$, where $\tau_{2}=\tau_{2}(C, \sigma)>0, r_{1}=r_{1}\left(r_{0}\right)<1$.

Proof. There is no loss of generality in assuming that $\theta=0$. Consider the function

$$
w\left(\rho e^{i \varphi}\right)=v\left(1-\rho e^{i \varphi}\right),
$$

which is subharmonic in the domain

$$
G=\left\{\zeta=\rho e^{i \varphi}: 0<\rho<1,0<\varphi<\pi / 4\right\} .
$$

We have

$$
w(s)<-\sigma \log \frac{e}{s}, s<\rho_{0}, \text { and } w\left(s e^{i \varphi}\right)<C \log \frac{e}{s}, s e^{i \varphi} \in G,
$$

and we need to prove that

$$
w\left(\rho e^{i \delta}\right)<-\frac{\sigma}{2} \log \frac{e}{\rho}, \quad \rho<\rho_{1} \text { for all } \delta \in\left(0, \rho \delta_{1}\right), \quad \delta_{1}=\delta_{1}(\sigma, C) .
$$

Consider an auxiliary function $u(\zeta)$ that is harmonic in the domain $R=\left\{\zeta=\rho e^{i \varphi}\right.$ : $1 / 4<|\rho|<1,0<\varphi<\pi / 4\}$ and has the boundary values

$$
u(\rho)=0, \quad \rho \in(1 / 4,1),\left.\quad u(\zeta)\right|_{\partial R \backslash(1 / 4,1)}=1 .
$$


Now we fix $\rho<\rho_{0} / 2$. By (3.3), we have

$$
w(\zeta)+\sigma \log \frac{e}{\rho} \leq(C+\sigma) u\left(\frac{\zeta}{2 \rho}\right) \log \frac{e}{\rho}+\sigma \log 2, \quad \zeta \in \partial(2 \rho R) .
$$

Therefore,

$$
w(\zeta)<-\sigma \log \frac{e}{\rho}+(C+\sigma) u\left(\frac{\zeta}{2 \rho}\right) \log \frac{e}{\rho}+\sigma \log 2, \quad \zeta \in 2 \rho R .
$$

In order to obtain (3.4), it remains to take $\zeta=\rho e^{i \varphi}$ and observe that $u\left(e^{i \varphi} / 2\right) \rightarrow 0$ as $\varphi \rightarrow 0$.

Lemmas 11, 2 and the Lusin-Privalov theorem imply that $\left|E_{+}(u)\right|=0$ for any $u \in \mathcal{K}$ and also $\left|D_{-}(f)\right|=0$ for any $f \in A^{-\infty}$.

\section{§4. Proof of Theorem 1}

In this section we prove Theorem [1. The first statement of this theorem is equivalent to the following claim.

Theorem 1(i). Suppose that $\lambda(t)=o\left(t|\log t|^{\omega}\right)$ as $t \rightarrow 0$, for every $\omega>0$. Then $H_{\lambda}\left(E^{+}(u)\right)=0$ for every $u \in \mathcal{K}$.

Proof. Fix $u \in \mathcal{K}$. Let

$$
E_{n}=\left\{e^{i \theta}: u\left(r e^{i \theta}\right) \geq \frac{1}{n} \log \frac{e}{1-r}, r \geq 1-\frac{1}{n}\right\} .
$$

Since $E_{+}(u)=\bigcup_{n} E_{n}$, it suffices to prove that $H_{\lambda}\left(E_{n}\right)=0$ for each $n$. We use the standard cone construction. For $e^{i \theta} \in \mathbf{T}$ and $a<1$, consider the Stolz angle $\Gamma_{\theta}^{a}=$ $\operatorname{conv}\left(e^{i \theta}, a \mathbf{D}\right)$, i.e., the convex hull of $e^{i \theta}$ and the disk of radius $a$. By Lemma 1 we can find $a>0$ such that $u(z) \geq \frac{1}{2 n} \log \frac{e}{1-|z|}$ for all $e^{i \theta} \in E_{n}$ and $z \in \Gamma_{a}^{\theta},|z|>1-\frac{1}{n}$.

Let

$$
\Omega=\bigcup_{\theta \in E_{n}} \Gamma_{a}^{\theta}
$$

The function $u$ is bounded from below on $\Omega$; let, say, $u \geq c_{0}$. Let $t<1$ be sufficiently close to 1 and

$$
\Omega_{t}=\Omega \cap t \mathbf{D}
$$

For an appropriate $b=b(a)$ we have

$$
\partial \Omega_{t} \cap t \mathbf{T}=t E_{n}^{b(1-t)}=\left\{t e^{i \theta}:\left|\theta-\theta_{0}\right|<b(1-t) \text { for some } e^{i \theta_{0}} \in E_{n}\right\} .
$$

Estimating the subharmonic function $u$ in the domain $\Omega_{t}, t>1-\frac{1}{n}$, in terms of harmonic measure, we obtain

$$
u(0) \geq c_{1}+\omega\left(0, t E_{n}^{b(1-t)}, \Omega_{t}\right) \frac{1}{2 n} \log \frac{e}{1-t} .
$$

The domains $\Omega_{t}$ have Lipschitz boundaries with Lipschitz constants bounded uniformly in $t$. By the Lavrentiev theorem (see, e.g., [6, Chapter VII, Theorem 4.3]), there exist $c$ and $\gamma$ such that, for each subarc $I \subset \partial \Omega_{t}$ and $A \subset I$, we have

$$
\frac{\omega\left(0, A, \Omega_{t}\right)}{\omega\left(0, I, \Omega_{t}\right)} \geq c\left(\frac{l(A)}{l(I)}\right)^{\gamma},
$$

where $l$ is the arc-length on $\partial \Omega_{t}$. In particular, by (4.1),

$$
l\left(t E_{n}^{b(1-t)}\right)^{\gamma} \leq c^{-1} l\left(\partial \Omega_{t}\right)^{\gamma} \omega\left(0, t E_{n}^{b(1-t)}, \Omega_{t}\right) \leq C\left(\log \frac{e}{1-t}\right)^{-1},
$$


where $C=C(n)$ does not depend on $t$. Hence, for all sufficiently small $\epsilon>0$ we have

$$
l\left(E_{n}^{\epsilon}\right) \leq C\left(\log \frac{b e}{\epsilon}\right)^{-1 / \gamma} .
$$

Therefore, $E_{n}$ can be covered by $N_{\epsilon}$ intervals of length $\epsilon$, with

$$
N_{\epsilon} \leq 2 \epsilon^{-1} C\left(\log \frac{b e}{\epsilon}\right)^{-1 / \gamma} \text {. }
$$

Then

$$
H_{\lambda}\left(E_{n}\right) \leq \liminf _{\epsilon \rightarrow 0} N_{\epsilon} \lambda(\epsilon) \leq \liminf _{\epsilon \rightarrow 0} 2 \epsilon^{-1} C\left(\log \frac{b e}{\epsilon}\right)^{-1 / \gamma} \lambda(\epsilon) .
$$

The condition on $\lambda$ implies that $H_{\lambda}\left(E_{n}\right)=0$ and we are done.

To prove the second part of Theorem 1 we repeat the argument for the subharmonic function $v(z)=\log |f(z)|$, using Lemma 2 instead of Lemma 1, and we replace the inequality (4.1) by the following estimate, valid for subharmonic functions:

$$
v(0) \leq \int_{\partial \Omega_{t}} v(z) d \omega\left(0, z, \Omega_{t}\right) \leq c_{1}-\omega\left(0, t E_{n}^{b(1-t)}, \Omega_{t}\right) \frac{1}{2 n} \log \frac{e}{1-t} .
$$

\section{$\S 5$. Sharpness of RESUlts}

First, we construct functions of class $\mathcal{K}$ with "large" sets of growth.

Lemma 3. For each integer $A \geq 2$, the series

$$
u(z)=\operatorname{Re} \sum_{k=0}^{\infty} A^{k} z^{2^{A^{k}}}
$$

converges in $\mathbf{D}$ and $|u(z)| \leq C \log \frac{e}{1-|z|}$.

Proof. We fix $z \in \mathbf{D}$ sufficiently close to the boundary and choose $N$ such that

$$
2^{-A^{N}} \geq 1-|z|>2^{-A^{N+1}} \text {. }
$$

Then

$$
-\log |z|=-\log (1-(1-|z|)) \geq 1-|z|>2^{-A^{N+1}},
$$

and for $n \geq N+1$ we have

$$
\frac{A^{n+1}|z|^{2^{A^{n+1}}}}{A^{n}|z|^{2^{A^{n}}}}=A|z|^{2^{A^{n+1}}-2^{A^{n}}} \leq A e^{-2^{-A^{N+1}}\left(2^{A^{n+1}}-2^{A^{n}}\right)}<\delta(A)<1
$$

with

$$
\lim _{A \rightarrow \infty} \delta(A)=0
$$

Therefore,

$$
\begin{aligned}
|u(z)| & \leq \sum_{n=0}^{N} A^{n}|z|^{2^{A^{n}}}+\sum_{n=N+1}^{\infty} A^{n}|z|^{2^{A^{n}}} \\
& \leq A^{N+1}+A^{N+1} \frac{1}{1-\delta(A)} \leq \frac{2}{1-\delta(A)} \frac{A}{\log 2} \log \frac{1}{1-|z|} .
\end{aligned}
$$


Proof of Proposition 1, For A sufficiently large, let

$$
f(z)=\exp \left(\sum_{k=1}^{\infty} A^{k} z^{2^{A^{k}}}\right)
$$

Then $u=\log |f|$ is a function as in the preceding lemma, so that both $f$ and $f^{-1}$ belong to $A^{-\infty}$. If for some $\phi$ we have $\cos \left(2^{A^{k}} \phi\right) \geq 1 / \sqrt{2}$ for each $k$, then $\operatorname{Re}\left(\left(r e^{i \phi}\right)^{2^{A^{k}}}\right) \geq$ $r^{2^{A^{k}}} / \sqrt{2}>0$. Taking $N$ as in (5.1), with $z=r e^{i \phi}$, we get

$$
u\left(r e^{i \phi}\right) \geq \frac{1}{2} A^{N} r^{2^{A^{N}}} \geq \frac{1}{8} A^{N} \geq \frac{1}{8 A \log 2} \log \frac{1}{1-r},
$$

whence $\phi \in E_{+}(u)$. Denote

$$
C_{j}=\bigcap_{k=0}^{j}\left\{\phi: \cos \left(2^{A^{k}} \phi\right) \geq 1 / \sqrt{2}\right\}, \quad C=\bigcap C_{j} .
$$

Then $C_{j}$ is the union of $N_{j}$ intervals of length $l_{j}=c 2^{-A^{j}}$, where $c$ is an absolute constant. We call them intervals of $j$ th generation. Each of them contains $k_{j+1}=c 2^{A^{j+1}-A^{j}}$ intervals of the next generation. So, it is easily seen that $N_{j}=c^{j} 2^{A^{j}}$. Now Theorem 3 with $\lambda(t)=t|\log t|^{\alpha}$ yields

$$
H_{\lambda}\left(E_{+}(u)\right) \geq H_{\lambda}(C) \geq \frac{c_{1}}{2 A^{\alpha}} \liminf _{j} c^{j} A^{\alpha j} .
$$

We choose $A$ such that $A^{\alpha}>c^{-1}$ and obtain $H_{\lambda}\left(E_{+}(u)\right)=+\infty$.

Next we construct an auxiliary harmonic function.

Lemma 4. There exist a function $h \in \operatorname{Harm}(\mathbf{D})$ and a positive $B$ such that $|h(z)| \leq$ $B|z|, z \in \mathbf{D}$, and

$$
\max _{1 / 6 \leq r \leq 1 / 3} h\left(r e^{i \theta}\right) \geq 1, \quad \theta \in[0,2 \pi) .
$$

Proof. Set $K=\left\{\frac{t+1}{6} e^{3 \pi t i}, 0 \leq t \leq 1\right\}$. Let $f$ be a function equal to 0 in a small neighborhood of 0 and to 2 in a small neighborhood of $K$. By the Runge theorem, we can approximate $f$ by a polynomial $g$ in such a way that $|g-f|<\frac{1}{3}$ on $K \cup\{0\}$. Then we can simply set $h=\operatorname{Re}(g-g(0))$.

Proposition 3 follows immediately from the following statement.

Lemma 5. If an integer $A$ is sufficiently large, then the series

$$
u(z)=\sum_{k=0}^{\infty} A^{k} h\left(z^{2^{A^{k}}}\right)
$$

converges in $\mathbf{D}$ to a function of class $\mathcal{K}$, and for some $d>0$ we have

$$
\limsup _{r \rightarrow 1} \frac{u\left(r e^{i \theta}\right)}{|\log (1-r)|} \geq d, \quad \theta \in[0,2 \pi) .
$$

Proof. We fix $z \in \mathbf{D}$ sufficiently close to the boundary and choose $N$ such that

$$
2^{-A^{N}} \geq 1-|z|>2^{-A^{N+1}} .
$$


By (5.2),

$$
\begin{aligned}
|u(z)| & \leq B \sum_{n=0}^{N} A^{n}|z|^{2^{A^{n}}}+B \sum_{n=N+1}^{\infty} A^{n}|z|^{2^{A^{n}}} \\
& \leq A^{N+1} B+A^{N+1} B \frac{1}{1-\delta(A)} \leq \frac{2}{1-\delta(A)} \frac{A B}{\log 2} \log \frac{1}{1-|z|} .
\end{aligned}
$$

The same estimate gives

$$
\left|u(z)-A^{N+1} h\left(z^{2^{A^{N+1}}}\right)\right| \leq \frac{A^{N+1} B}{A-1}+A^{N+1} B \sum_{k \geq 1} \delta(A)^{k} \leq \frac{A^{N+1}}{2}
$$

for large $A$.

Finally, given $\theta \in[0,2 \pi)$, we construct a sequence of points $\left\{w_{N}=\left|w_{N}\right| e^{i \theta}\right\}$ at which $u$ has extremal growth.

Let $r_{N}(\theta) \in\left[\frac{1}{6}, \frac{1}{3}\right]$ be such that

$$
h\left(r_{N}(\theta) e^{i \theta \cdot 2^{A^{N+1}}}\right) \geq 1,
$$

and let $w_{N}=r_{N}(\theta)^{2^{-A^{N+1}}} e^{i \theta}$. Then

$$
2^{-A^{N}} \geq 1-\left|w_{N}\right|>2^{-A^{N+1}}
$$

and

$$
\begin{aligned}
u\left(w_{N}\right) & \geq A^{N+1} h\left(w_{N}^{2^{A^{N+1}}}\right)-\frac{A^{N+1}}{2}=A^{N+1} h\left(r_{N}(\theta) e^{i \theta \cdot 2^{A^{N+1}}}\right)-\frac{A^{N+1}}{2} \\
& \geq \frac{A^{N+1}}{2} \geq c \log \frac{1}{1-\left|w_{N}\right|} .
\end{aligned}
$$

Proof of Proposition 2, Now, let $\mu>1$, let an integer $\beta>1$ satisfy (2.4), and let $f_{\mu, \beta}$ be the corresponding Horowitz function; see (2.3). The zeros of $f_{\mu, \beta}$ are of the form

$$
z_{k, j}=\rho_{k} e^{i \pi \beta^{-k}} e^{2 i \pi j \beta^{-k}}, \quad k=1,2, \ldots, j=0,1, \ldots, \beta^{k}-1,
$$

where $\rho_{k}=\mu^{-\beta^{-k}}$.

We shall construct a sequence $\left\{\epsilon_{k}\right\}$ such that $\epsilon_{k} \searrow 0$,

$$
\sum_{k=1}^{\infty} \beta^{k} \epsilon_{k}<\infty
$$

and, for some $a>0$,

$$
\left|f_{\mu, \beta}(z)\right| \geq \frac{\text { Const }}{(1-|z|)^{a}} \quad \text { for } \quad z \notin \bigcup_{k, j} D_{k, j}
$$

where $D_{k, j}=\left\{z \in \mathbf{D}:\left|z-z_{k, j}\right|<\epsilon_{k}\right\}$. Since $\rho_{k}=\mu^{-\beta^{-k}}$, the disks $D_{k, j}$ are contained in the open unit disk; by (5.4), the sum of the lengths of the projections of $\bigcup_{j} D_{k, j}$ to $\mathbf{T}$ is finite. Now Proposition 2 follows readily.

Consider the circle $|z|=r<1$ and choose an integer $m$ such that

$$
r^{\beta^{m}} \geq \mu^{-\sqrt{\beta}}>r^{\beta^{m+1}}
$$

then

$$
\mu r^{\beta^{m-1}} \geq \mu^{1-1 / \sqrt{\beta}}>1 \text { and } \mu r^{\beta^{m+1}}<\mu^{1-\sqrt{\beta}}<1 \text {. }
$$


Also, relation (5.6) yields

$$
\left|m-\frac{1}{\log \beta} \log \frac{1}{1-r}\right|<C,
$$

where the constant $C$ does not depend on $r$. We may assume that $m>1$. We have

$$
f(z)=\underbrace{\prod_{k=1}^{m-1}\left(1+\mu z^{\beta^{k}}\right)}_{P_{m}(z)}\left(1+\mu z^{\beta^{m}}\right) \underbrace{\prod_{k=m+1}^{\infty}\left(1+\mu z^{\beta^{k}}\right)}_{R_{m}(z)} .
$$

The first factor is uniformly large on the circle $|z|=r$ :

$$
\log \left|P_{m}(z)\right| \geq \sum_{k=1}^{m-1} \log \left(\mu r^{\beta^{k}}-1\right)=\sum_{k=1}^{m-1}\left(\log \mu+\beta^{k} \log r\right)+\sum_{k=1}^{m-1} \log \left(1-\frac{1}{\mu r^{\beta^{k}}}\right) .
$$

We get

$$
\sum_{k=1}^{m-1}\left(\log \mu+\beta^{k} \log r\right) \geq(m-1) \log \mu+\beta^{m} \log r \geq(m-1) \log \mu-\sqrt{\beta} \log \mu
$$

and

$$
\sum_{k=1}^{m-1} \log \left(1-\frac{1}{\mu r^{\beta^{k}}}\right) \geq \sum_{k=1}^{m-1} \log \left(1-\frac{1}{\mu r^{\beta^{m-1}}}\right) \geq(m-1) \log \left(1-\frac{1}{\mu^{1-1 / \sqrt{\beta}}}\right) .
$$

Now relation (2.4) yields

$$
\log \left|P_{m}(z)\right| \geq(m-1)(\log \mu-1)-\sqrt{\beta} \log \mu,
$$

and, by (5.7),

$$
\left|P_{m}(z)\right| \geq \frac{\text { Const }}{(1-|z|)^{a}}, \quad|z|=r, \quad a=\frac{\log \mu-1}{\log \beta} .
$$

We apply the inequality $\log (1-x) \geq-\alpha x, x \leq 1-\alpha^{-1}$, in order to prove that the third factor in (5.8) is separated away from zero whenever $|z|=r$ :

$$
\log \left|R_{m}(z)\right| \geq \sum_{k=m+1}^{\infty} \log \left(1-\mu|z|^{\beta^{k}}\right) \geq-\alpha \sum_{k=m+1}^{\infty} \mu|z|^{\beta^{k}} \geq c(\mu, \beta)
$$

if we take $\alpha=\left(1-\mu^{1-\sqrt{\beta}}\right)^{-1}$, say.

The second factor in (5.8) vanishes at the points $\left\{z_{m, j}\right\}, j=0,1, \ldots, \beta^{m}-1$. Fix $q<1$ and let $\epsilon_{m}=q^{m} \beta^{-m}$. Then condition (5.4) is fulfilled. It is also straightforward that

$$
\left|1+\mu z^{\beta^{m}}\right| \geq c q^{m}
$$

if $\left|z-z_{m, j}\right|=\epsilon_{m}$ for some $j$. Then the minimum principle implies the same inequality whenever $\operatorname{dist}\left(z,\left\{z_{m, j}\right\}_{j}\right)>\epsilon_{m}$.

Now from (5.7) it follows that, for any $a^{\prime}<a$, one can choose $q$ sufficiently close to 1 and such that

$$
\left|f_{\mu, \beta}(z)\right| \geq \frac{\text { Const }}{(1-|z|)^{a^{\prime}}} \quad \text { for } z \notin \bigcup_{k, j} D_{k, j} .
$$

This completes the proof of Proposition 2 , 


\section{$\S 6$. Positive harmonic Functions}

In this section we prove Theorem 2 First, we check that, for any positive function $u \in \mathcal{K}$, the set

$$
F_{+}(u)=\left\{\theta \in[0,2 \pi): \limsup _{r \rightarrow 1} \frac{u\left(r e^{i \theta}\right)}{|\log (1-r)|}>0\right\}
$$

is a countable union of sets with finite $H_{\lambda}$-measure, with the measuring function $\lambda(t)=$ $t|\log t|$. This implies the first statement of Theorem 2 .

Let

$$
F_{n}=\left\{\theta \in[0,2 \pi): \limsup _{r \rightarrow 1} \frac{u\left(r e^{i \theta}\right)}{|\log (1-r)|} \geq \frac{2}{n}\right\} .
$$

It suffices to prove that $H_{\lambda}\left(F_{n}\right)<\infty$ for all $n$.

Since the function $u$ is positive and harmonic, it is the Poisson integral of a finite measure $\mu$ on $\mathbf{T}$. Since $u \in \mathcal{K}$, we have

$$
\mu(I) \leq C|I| \log \frac{e}{|I|}
$$

for any $\operatorname{arc} I$ on the unit circle (see [10]).

In what follows we denote

$$
\mu(\alpha, \beta)=\mu\left(\left\{e^{i \varphi}: \alpha \leq \varphi<\beta\right\}\right) .
$$

Lemma 6. For each $n$ and each $\theta \in F_{n}$, there exists a decreasing sequence $\left\{\Delta_{j}\right\}, \Delta_{j} \rightarrow 0$ as $j \rightarrow \infty$, that satisfies

$$
\mu\left(\theta-\Delta_{j}, \theta+\Delta_{j}\right) \geq k\left(10 \Delta_{j} \log \frac{1}{10 \Delta_{j}}\right)
$$

with some $k>0$ depending on $C$ and $n$ only.

Suppose this lemma is already proved. For each $\epsilon>0$ we can cover $F_{n}$ by intervals $I$ with centers on $F_{n}$, of length less than $\epsilon$, and satisfying $\mu(I) \geq k|5 I||\log | 5 I||$, where $5 I$ is the interval concentric with $I$ of length 5 times that of $I$. By the Vitali lemma (see, for example, [7, page 2]), we can find a subfamily $I_{j}$ of disjoint intervals such that $F_{n} \subset \bigcup_{j} 5 I_{j}$. We obtain

$$
\sum_{j}\left|5 I_{j}\right||\log | 5 I_{j}|| \leq \frac{1}{k} \sum_{j} \mu\left(I_{j}\right) \leq \frac{1}{k} \mu(\mathbf{T}),
$$

which yields $H_{\lambda}\left(F_{n}\right) \leq \frac{1}{k} \mu(\mathbf{T})<+\infty$.

Proof of Lemma 6. For $\theta \in F_{n}$ there exists a sequence $\left\{r_{j}\right\}_{1}^{\infty}$ such that $r_{j} \nearrow 1$ and

$$
\frac{1}{n} \log \frac{1}{1-r_{j}} \leq u\left(r_{j} e^{i \theta}\right)=\int_{-\pi}^{\pi} P\left(r_{j} e^{i \phi}\right) d \mu(\theta-\phi) .
$$

Let $a, A$ be two constants such that $0<a<A$ (their values will be determined below), and let $\delta_{j}=a\left(1-r_{j}\right), \Delta_{j}=A\left(1-r_{j}\right)$. By choosing $a$ sufficiently small and using (6.1), we can achieve

$$
\int_{-\delta_{j}}^{\delta_{j}} P\left(r_{j} e^{i \phi}\right) d \mu(\theta-\phi) \leq \frac{1}{10 n} \log \frac{1}{1-r_{j}}, \quad j>j_{0}
$$

Next, let

$$
Q\left(r e^{i \phi}\right)=-\partial_{\phi} P\left(r e^{i \phi}\right)=\frac{1}{2 \pi} \frac{2 r\left(1-r^{2}\right) \sin \phi}{\left(1-2 r \cos \phi+r^{2}\right)^{2}}
$$


be the angular derivative of the Poisson kernel. Then we have

$$
\begin{aligned}
& \int_{\delta_{j}<|\phi| \leq \pi} P\left(r_{j} e^{i \phi}\right) d \mu(\theta-\phi) \leq \mu(\mathbf{T})+\int_{\delta_{j}}^{\pi} \mu(\theta-\phi, \theta+\phi) Q\left(r_{j} e^{i \phi}\right) d \phi \\
& =\mu(\mathbf{T})+\int_{\delta_{j}}^{\Delta_{j}} \mu(\theta-\phi, \theta+\phi) Q\left(r_{j} e^{i \phi}\right) d \phi+\int_{\Delta_{j}}^{\pi} \mu(\theta-\phi, \theta+\phi) Q\left(r_{j} e^{i \phi}\right) d \phi .
\end{aligned}
$$

Moreover,

$$
\begin{aligned}
& \int_{\Delta_{j}}^{\pi} \mu(\theta-\phi, \theta+\phi) Q\left(r_{j} e^{i \phi}\right) d \phi \leq C \log \frac{e}{2 \Delta_{j}} \int_{\Delta_{j}}^{\pi} 2 \phi Q\left(r_{j} e^{i \phi}\right) d \phi \\
& \quad \leq 2 C \log \frac{1}{1-r_{j}}\left(A\left(1-r_{j}\right) P\left(r_{j} e^{i A\left(1-r_{j}\right)}\right)+\int_{A\left(1-r_{j}\right)}^{\pi} P\left(r_{j} e^{i \phi}\right) d \phi\right) .
\end{aligned}
$$

Taking $A$ sufficiently large, we obtain

$$
\int_{\Delta_{j}}^{\pi} \mu(\theta-\phi, \theta+\phi) Q\left(r_{j} e^{i \phi}\right) d \phi \leq \frac{1}{10 n} \log \frac{1}{1-r_{j}}, \quad j>j_{0} .
$$

Now, from (6.3), (6.4), (6.5), and (6.6) it follows that

$$
\int_{\delta_{j}}^{\Delta_{j}} \mu(\theta-\phi, \theta+\phi) Q\left(r_{j} e^{i \phi}\right) d \phi>\frac{1}{5 n} \log \frac{1}{1-r_{j}}, \quad j>j_{0} .
$$

Integration by parts gives

$$
\int_{\delta_{j}}^{\Delta_{j}} \phi Q\left(r_{j} e^{i \phi}\right) d \phi \leq \int_{0}^{\pi} \phi Q\left(r_{j} e^{i \phi}\right) d \phi \leq \int_{0}^{\pi} P\left(r_{j} e^{i \phi}\right) d \phi=\frac{1}{2} .
$$

Together with (6.7), this implies that

$$
\int_{\delta_{j}}^{\Delta_{j}} \mu(\theta-\phi, \theta+\phi) Q\left(r_{j} e^{i \phi}\right) d \phi>\frac{1}{5 n} \log \frac{1}{1-r_{j}} \int_{\delta_{j}}^{\Delta_{j}} \phi Q\left(r_{j} e^{i \phi}\right) d \phi, \quad j>j_{0} .
$$

Therefore, for each $j>j_{0}$ there exists $\phi_{j} \in\left(\delta_{j}, \Delta_{j}\right)$ such that

$$
\mu\left(\theta-\phi_{j}, \theta+\phi_{j}\right)>\frac{\phi_{j}}{5 n} \log \frac{1}{1-r_{j}} .
$$

The desired estimate (6.2) follows.

To complete the proof of Theorem 2, we need to construct a positive harmonic function $u \in \mathcal{K}$ with $H_{\lambda}\left(E_{+}(u)\right)=\infty$, where $\lambda(t)=t|\log t|$. Taking then its harmonic conjugate $\widetilde{u}$, we arrive at the desired function $f=\exp (u+i \widetilde{u})$. Clearly $D_{+}(f)=E_{+}(u), f \in A^{-\infty}$, and $|f| \geq 1$.

First, we construct a function $v \in \mathcal{K}$ such that $H_{\lambda}\left(E_{+}(v)\right)>0$. We use a Cantor-type construction.

Let $C_{1}$ be the union of two opposite quarters of the circle. By induction, we construct sets $C_{k} \subset C_{k-1}$ such that $C_{k}$ consists of $2^{2^{k}-k}$ closed arcs of length $2^{1-2^{k}} \pi$ each. To obtain $C_{k}$, we divide each of the arcs of $C_{k-1}$ into $2^{2^{k-1}}$ equal subarcs and choose each second of them for $C_{k}$. Denote $C=\bigcap C_{k}$ and consider the measures $d \mu_{k}=2{ }^{k} \chi\left(C_{k}\right) d t$, where $\chi\left(C_{k}\right)$ is the characteristic function of $C_{k}$.

Lemma 7. The sequence $\left\{\mu_{k}\right\}$ converges weakly to a measure $\mu_{0}$, and $v=P * \mu_{0} \in \mathcal{K}$. Moreover, $C \subset E_{+}(v)$ and $H_{\lambda}(C)>0$. 
Proof. We note that $\mu_{k}(\mathbf{T})=2 \pi$ for each $k$. Also, for each arc $I$ with endpoints of the form $\exp \left(2 \pi m i 2^{-2^{s}}\right)$, where $m$ is an integer, the limit $\mu_{k}(I)$ as $k \rightarrow \infty$ exists, simply because all values $\mu_{k}(I)$ are the same when $k>s$. Now, each continuous function on the circle can be uniformly approximated by linear combinations of the characteristic functions of such dyadic arcs. Thus, for each continuous function $f$, the limit

$$
\lim _{k \rightarrow \infty} \int_{\mathbf{T}} f d \mu_{k}
$$

exists, and the $\mu_{k}$ converge weakly to some positive measure $\mu_{0}$.

In order to prove that $v=P * \mu_{0} \in \mathcal{K}$, it suffices to check that

$$
\mu_{0}(J) \leq \operatorname{const}|J| \log \frac{1}{|J|}
$$

for each $\operatorname{arc} J \subset \mathbf{T}$, and then to use again the results of [10].

Choose $s$ such that $2^{-2^{s}} 2 \pi<|J| \leq 2^{-2^{s-1}} 2 \pi$ and take an arc $J_{0} \supset J$ with endpoints of the form $\exp \left(2 \pi m i 2^{-2^{s}}\right)$ with $m \in \mathbb{Z}$ and such that $\left|J_{0}\right|<3|J|$. We obtain

$$
\mu_{k}(J) \leq \mu_{k}\left(J_{0}\right)=\mu_{s}\left(J_{0}\right) \leq 2^{s}\left|J_{0}\right|<6|J||\log | J||,
$$

which is the desired inequality.

Now we check that $C \subset E_{+}(v)$. We have

$$
v\left(r e^{i \alpha}\right)=\int_{-\pi}^{\pi} P\left(r e^{i \phi}\right) d \mu_{0}(\alpha-\phi) \geq \int_{0}^{\pi} \mu_{0}(\alpha-\phi, \alpha+\phi) Q\left(r e^{i \phi}\right) d \phi .
$$

Let $\alpha \in \bigcap C_{k}=C$, and let $2^{k-1} \leq|\log (1-r)|<2^{k}$. Then $\mu_{0}(\alpha-\phi, \alpha+\phi) \geq c 2^{k} \phi$ for $\phi<1-r$, and

$$
v\left(r e^{i \alpha}\right) \geq \int_{0}^{1-r} \mu_{0}(\alpha-\phi, \alpha+\phi) Q\left(r e^{i \phi}\right) d \phi \geq c 2^{k} \int_{0}^{1-r} \phi Q\left(r e^{i \phi}\right) d \phi \geq c_{1} 2^{k}
$$

for $r>r_{0}$. Thus, $C=\bigcap C_{k} \subset E_{+}(v)$. Recall that $C_{k}$ is the union of $2^{2^{k}-k}$ arcs of length $2 \pi 2^{-2^{k}}$ and $C$ is a set of the type described in Theorem 3. For $\lambda(t)=t|\log t|$ the theorem gives $H_{\lambda}(C) \geq c>0$.

Finally, we construct a sequence of measures $\mu^{(n)}$ and sets $C^{(n)}$ such that $v^{(n)}=$ $P * \mu^{(n)}$ is in $\mathcal{K}, E_{+}\left(v^{(n)}\right) \supset C^{(n)}$, and $H_{\lambda}\left(C^{(n)}\right) \rightarrow \infty$ as $n \rightarrow \infty$.

The construction of $C^{(n)}$ is as follows. Let $C_{1}^{(n)}$ be the union of $2^{n} \operatorname{arcs}$ of length $2 \pi 2^{-n-1}$ (we split the circle into $2^{n+1}$ equal arcs and take every other one), and let $\mu_{1}^{(n)}=2^{-n+1} \chi\left(C_{1}^{(n)}\right)$. Let $C_{k}^{(n)}$ be the union of $2^{2^{k-1}(n+1)-k}$ arcs of length $2 \pi 2^{-2^{k-1}(n+1)}$; then we split each arc into equal arcs of length $2 \pi 2^{-2^{k}(n+1)}$ and take every other one of them to form $C_{k+1}$. We define also $\mu_{k}^{(n)}=2^{-n+k} \chi\left(C_{k}^{(n)}\right)$. As before, the sequence of measures $\mu_{k}^{(n)}$ converges to a singular measure $\mu^{(n)}$ such that

$$
\mu^{(n)}(J) \leq \text { const } \cdot 2^{-n} \cdot|J| \log \frac{1}{|J|}
$$

for every $n \geq 1$ and every arc $J \subset \mathbf{T}$. Then $v^{(n)}=P * \mu^{(n)} \in \mathcal{K}, u=\sum_{n \geq 1} v^{(n)} \in \mathcal{K}$, and $E_{+}\left(v^{(n)}\right) \supset C^{(n)}$. Theorem 3 shows that $H_{\lambda}\left(C^{(n)}\right) \geq c n$, and, hence, $H_{\lambda}\left(E_{+}(u)\right)=\infty$. 


\section{$\S 7$. HausdorfF measure of Cantor sets}

In this section we prove Theorem 3 . The left-hand side inequality in (2.6) is straightforward.

We say that $C_{s}$ is the set of $s$ th generation, and the intervals $I_{i}^{(s)}$ of length $l_{s}$ that constitute $C_{s}$ are the intervals of $s$ th generation. The set of all these intervals will be denoted by $\mathcal{I}_{s}$.

Let $\left\{J_{j}\right\}$ be a finite covering of $C$ by intervals of length less than $l_{s}$. We split the set $\left\{J_{j}\right\}$ into finitely many groups $\mathcal{A}_{s}, \mathcal{A}_{s+1}, \ldots, \mathcal{A}_{m}$, where

$$
\mathcal{A}_{p}=\left\{J_{j}: l_{p+1} \leq\left|J_{j}\right|<l_{p}\right\} .
$$

Some of these groups may be empty. Put

$$
\mathcal{M}_{s+1}=\left\{I \in \mathcal{I}_{s+1}: I \cap\left(\bigcup_{J \in \mathcal{A}_{s}} J\right) \neq \varnothing\right\} \text {, and } M_{s+1}=\# \mathcal{M}_{s+1} \text {. }
$$

We have

$$
M_{s+1} \leq \sum_{J \in \mathcal{A}_{s}}\left(\frac{|J|}{l_{s+1}}+1\right) \leq 2 \sum_{J \in \mathcal{A}_{s}} \frac{|J|}{l_{s+1}}
$$

Put $\mathcal{R}_{s+1}=\mathcal{I}_{s+1} \backslash \mathcal{M}_{s+1}$ and $R_{s+1}=\# \mathcal{R}_{s+1}=N_{s+1}-M_{s+1}$. We have $R_{s+1}$ intervals from $\mathcal{I}_{s+1}$ that do not intersect intervals from $\mathcal{A}_{s}$.

We continue the procedure. The number of all intervals from $\mathcal{I}_{s+2}$ that are contained in $\bigcup_{I \in \mathcal{R}_{s+1}} I$ is $k_{s+1} R_{s+1}$. Let $M_{s+2}$ be the number of such intervals that intersect $\bigcup_{J \in \mathcal{A}_{s+1}} J$, and let $R_{s+2}=k_{s+1} R_{s+1}-M_{s+2}$ be the number of remaining intervals.

After several steps we have $R_{q}$ intervals from $\mathcal{I}_{q}$ that intersect no interval from $\mathcal{A}_{s} \cup$ $\mathcal{A}_{s+1} \cup \cdots \cup \mathcal{A}_{q-1}$. Then we have $k_{q} R_{q}$ intervals in $\mathcal{I}_{q+1}$ that intersect no interval from $\mathcal{A}_{s} \cup \mathcal{A}_{s+1} \cup \cdots \cup \mathcal{A}_{q-1}$, and $M_{q+1}$ of them intersect intervals from $\mathcal{A}_{q}$, where

$$
M_{q+1} \leq 2 \sum_{J \in \mathcal{A}_{q}} \frac{|J|}{l_{q+1}} .
$$

Next we define $R_{q+1}=k_{q} R_{q}-M_{q+1}$. By induction,

$$
R_{q+1}=N_{q+1}-\sum_{r=s+1}^{q+1} \frac{M_{r}}{N_{r}} N_{q+1} .
$$

If $R_{m+1}>0$, then we can find a point in $C$ that is not covered by the intervals from $\mathcal{A}_{s} \cup \cdots \cup \mathcal{A}_{m}$. Thus, $R_{m+1}=0$, and we get

$$
\sum_{r=s+1}^{m+1} \frac{M_{r}}{N_{r}}=1
$$

Set $b_{s}=\inf _{q \geq s} N_{q} \lambda\left(l_{q}\right)$. Now we use (2.5), (17.1), (17.2) to estimate the sum of $\lambda\left(\left|J_{j}\right|\right)$ :

$$
\begin{aligned}
\sum_{j} \lambda\left(\left|J_{j}\right|\right) & =\sum_{p=s}^{m} \sum_{J \in \mathcal{A}_{p}} \lambda(|J|) \geq a \sum_{p=s}^{m} \sum_{J \in \mathcal{A}_{p}} \lambda\left(l_{p+1}\right) \frac{|J|}{l_{p+1}} \\
& \geq a b_{s} \sum_{p=s}^{m} \frac{1}{N_{p+1}} \sum_{J \in \mathcal{A}_{p}} \frac{|J|}{l_{p+1}} \geq \frac{a b_{s}}{2} \sum_{p=s}^{m} \frac{M_{p+1}}{N_{p+1}}=\frac{a}{2} b_{s}
\end{aligned}
$$

for any finite cover of $C$ with $\left|J_{j}\right|<l_{s}$. This shows that

$$
H_{\lambda}(C) \geq \frac{a}{2} \liminf _{s \rightarrow \infty} N_{s} \lambda\left(l_{s}\right) .
$$




\section{ACKNOWLEDGMENTS}

This work was done when Yu. L. and E. M. visited the Universities of Aix-Marseille and Paul Sabatier. They thank the Universities for their hospitality and support.

\section{REFERENCES}

[1] F. Bagemihl and W. Seidel, Some boundary properties of analytic functions, Math. Z. 61 (1954), 186-199. MR0065643(16:460d)

[2] A. Borichev, On the minimum of harmonic functions, J. Anal. Math. 89 (2003), 199-212. MR 1981918 (2004h:31001)

[3] A. Borichev and Yu. Lyubarskii, Uniqueness theorems for Korenblum type spaces, J. Anal. Math. 103 (2007), 307-329. MR2373272 (2009a:30005)

[4] C. Cabrelli, F. Mendivil, U. Molter, and R. Shonkwiler, On the Hausdorff h-measure of Cantor sets, Pacific J. Math. 217 (2004), no. 1, 45-59. MR.2105765 (2005h:28013)

[5] V. Eŭderman, On a comparison between the Hausdorff measure and capacity, Algebra i Analiz 3 (1991), no. 6, 173-188; English transl., St. Petersburg Math. J. 3 (1992), no. 6, 1367-1381. MR:1167683 (93m:31007)

[6] J. Garnett and D. Marshall, Harmonic measure, New Math. Monogr., vol. 2, Cambridge Univ. Press, Cambridge, 2005, xvi+571 pp. MR2150803 (2006g:31002)

[7] J. Heinonen, Lectures on analysis on metric spaces, Universitext, Springer-Verlag, New York, 2001. MR1800917 (2002c:30028)

[8] C. Horowitz, Zeros of functions in the Bergman spaces, Duke Math. J. 41 (1974), 693-710. MR0357747(50:10215)

[9] J.-P. Kahane and Y. Katznelson, Sur le comportement radial des fonctions analytiques, C. R. Acad. Sci. Paris Sér. A-B 272 (1971), A718-A719. MR0277721 (43:3454)

[10] B. Korenblum, An extension of the Nevanlinna theory, Acta Math. 135 (1975), no. 3-4, 187-219. MR 0425124(54:13081)

[11] N. K. Nikol'skiǐ, Selected problems of weighted approximation and spectral analysis, Trudy Mat. Inst. Steklov. 120 (1974), 272 pp.; English transl., Proc. Steklov Inst. Math. 1976, 276 pp. MR0467269 (57:133a) MR0467270 (57:133b)

[12] I. I. Privalov, Boundary properties of analytic functions, Gostekhizdat, Moscow-Leningrad, 1950. (Russian) MR0047765 (13:926h)

[13] K. Seip, On Korenblum's density condition for the zero sequences of $A^{-\infty}$, J. Anal. Math. 67 (1995), 307-322. MR1383499 (97c:30044)

Université Aix-Marseille, 39 Rue Joliot Curie, 13453, Marseille Cedex 13, France

E-mail address: borichev@cmi.univ-mrs.fr

Department of Mathematical Sciences, Norwegian University of Science and Technology, NO-7491, TrondheIm, NorWAY

E-mail address: yura@math.ntnu.no

Department of Mathematical Sciences, Norwegian University of Science and Technology, NO-7491, TrondheIM, Norway

E-mail address: eugenia@math.ntnu.no

Université Paul Sabatier, 31062, Touluose Cedex 9, France

E-mail address: pthomas@math.univ-toulouse.fr

Received 27/MAR/2009

Originally published in English 